\title{
Signalling components involved in contraction-inducible substrate uptake into cardiac myocytes
}

\author{
Joost J. F. P. Luiken ${ }^{1 *}$, Susan L. M. Coort ${ }^{1}$, Debby P. Y. Koonen ${ }^{1}$, Arend Bonen ${ }^{2}$ \\ and Jan F. C. Glatz \\ ${ }^{1}$ Department of Molecular Genetics, Cardiovascular Research Institute Maastricht (CARIM), Maastricht University, \\ NL-6200 MD Maastricht, The Netherlands \\ ${ }^{2}$ Department of Human Biology and Nutritional Sciences, University of Guelph, Guelph, Ontario N1G 2W1, Canada
}

\begin{abstract}
Glucose and long-chain fatty acids (LCFA) are two major substrates used by heart and skeletal muscle to support contractile activity. In quiescent cardiac myocytes a substantial portion of the glucose transporter GLUT4 and the putative LCFA transporter fatty acid translocase (FAT)/CD36 are stored in intracellular compartments. Induction of cellular contraction by electrical stimulation results in enhanced uptake of both glucose and LCFA through translocation of GLUT4 and FAT/CD36 respectively to the sarcolemma. The involvement of protein kinase A, AMP-activated protein kinase (AMPK), protein kinase $\mathrm{C}$ (PKC) isoforms and the extracellular signal-regulated kinases was evaluated in cardiac myocytes as candidate signalling enzymes involved in recruiting these transporters in response to contraction. The collected evidence excluded the involvement of PKA and implicated an important role for AMPK and for one (or more) PKC isoform(s) in contraction-induced translocation of both GLUT4 and FAT/CD36. The unravelling of further components along this contraction pathway can provide valuable information on the coordinated regulation of the uptake of glucose and of LCFA by an increase in mechanical activity of heart and skeletal muscle.
\end{abstract}

Isolated cardiac myocytes are an excellent system in which to study the signalling and metabolic processes that take place during and as a result of muscle contractions, as will be shown in the present review. In general, single-cell suspensions are easily accessible to pharmacologicallyactive compounds, while at the same time the extracellular environment is well controlled and vascular factors are eliminated. The standard procedure for obtaining singlecell suspensions from the heart is a retrograde perfusion with collagenase. Unfortunately, it is virtually impossible to obtain single cells from skeletal muscle for metabolic studies, as the muscle fibre is long and fragile because it originates from multiple cell-fusion processes. The next best approach for in vitro studies in skeletal muscle research is the isolated muscle strip. When muscle strips are removed from the hindlimb together with their tendons it is possible to impose contractions in these strips through electric stimulation. However, the muscle strip comprises several cell layers, which results in serious diffusion problems for compounds (substrates, agents) that are added to the medium. Despite subtle differences, skeletal myocytes and cardiac myocytes share many features of the regulation of cell function, including signalling and metabolism. The availability of an experimental system that induces contractions in cardiac myocytes in suspension makes it possible to investigate the signalling processes that are induced by these contractions, while at the same time the cardiac myocytes can be exposed to selected compounds exerting specific pharmacological effects. It is then expected that the findings in contracting cardiac myocytes can be extrapolated to contracting skeletal muscle.

\section{Cardiac myocytes as a model for the study of substrate uptake}

Contractions are a permanent manifestation of cardiac myocytes in vivo, and necessary for the maintenance of

\footnotetext{
Abbreviations: AICAR, 5-aminoimidazole-4-carboxamide-1- $\beta$-D-riboside; AMPK, AMP-activated protein kinase; FAT, fatty acid translocase; LCFA, long-chain fatty acids; PMA, phorbol 12-myristate 13-acetate; PKA, PKC, protein kinase A and C respectively.

*Corresponding author: Joost J. F. P. Luiken, fax +31 43388 4574, email j.luiken@ gen.unimaas.nl
} 
cardiac pump function. However, when they are isolated and subsequently incubated the trigger for controlled contractions is not present. This factor causes the metabolism of isolated cardiac myocytes in suspension to be much slower than that in vivo, as exemplified by the rate of $\mathrm{O}_{2}$ consumption, which is only approximately $10 \%$ of that of the intact working heart (Luiken et al. 2001). Accordingly, the demands of isolated cardiac myocytes for fuel, predominantly glucose and long-chain fatty acids (LCFA), will be markedly lower than that in the in vivo situation.

Recently, an experimental system was developed to perform metabolic measurements with suspensions of electrically-stimulated cardiac myocytes, thereby increasing their rate of $\mathrm{O}_{2}$ consumption up to approximately $50 \%$ of that in vivo, depending on the applied frequency. As a result, the uptake of both glucose and LCFA increases in proportion to the pulse frequency. For instance, for $4 \mathrm{~Hz}$ contractions the rates of uptake of both substrates are similarly increased to approximately $1 \cdot 5$-fold within minutes (Luiken et al. 2001). This similarity in acute response in the uptake process of both substrates might seem paradoxical. On the one hand, the heart is an omnivorous organ that can utilize both glucose and LCFA to fulfill the increased demands for fuel. Furthermore, for both substrates the concentration gradient across the sarcolemma is the driving force for uptake (Kones \& Phillips, 1975; van der Vusse et al. 1992). Specifically, the rapid metabolic trapping of both substrates, in the case of glucose its phosphorylation to glucose-6-phosphate and in the case of LCFA its esterification with CoA to acyl-CoA, serves to maintain a low substrate concentration intracellularly. On the other hand, the difference in chemical properties between glucose and LCFA suggests that these substrates traverse the phospholipid bilayer of the sarcolemma through entirely different mechanisms (Hamilton, 1998) and, therefore, would not be expected to show a similar response to increased contractile activity. In hindsight, the conundrum is explained by the fact that the uptake of both substrates has been proved to obey the laws of facilitated diffusion, and also that the mechanism by which contractions induce uptake of both substrates by cardiac myocytes appears to be similar, as first shown in skeletal muscle (Bonen et al. 2002; Glatz et al. 2002).

\section{Mechanism of uptake of glucose and long-chain fatty acids in heart and skeletal muscle}

In skeletal muscle the molecular mechanism by which short-term contractions induce the uptake of glucose are well understood and involve a vesicle-mediated translocation of GLUT4 from an endosomal subcompartment to the sarcolemma (Cushman et al. 1998; Zorzano et al. 2000; Ploug \& Ralston, 2002). This mechanism of contractioninduced GLUT4 translocation has subsequently been confirmed also in the heart (Till et al. 1997). In relation to the molecular mechanism by which short-term contractions induce cardiac LCFA uptake, an initial clue was provided by observations with sulfo- $N$-succinimidyloleate, which specifically inhibits FAT/CD36. This compound not only reduced LCFA uptake under non-stimulated conditions by approximately $50 \%$, but it was also able to completely block the contraction-induced increase in LCFA uptake by isolated cardiac myocytes (Luiken et al. 2001). This finding strongly suggests that FAT/CD36 is involved in the acute response of cardiac myocytes to an increase in workload. The increased flux through FAT/ CD36 during cardiac myocyte contractions can be a result of either (1) an increase in sarcolemmal abundance of this protein or (2) an increase in its intrinsic transport activity. In similar studies with short-term electrically-stimulated rat skeletal muscle, in which LCFA uptake was studied as a function of the exogenous LCFA concentration, it was found that following contractions the apparent maximum velocity for LCFA uptake was increased while there was no change in the apparent $K_{m}$ (Bonen et al. 2000). This observation suggests that an increased sarcolemmal abundance is the most likely mechanism to explain the higher LCFA uptake rate. As changes in FAT/CD36 protein expression are not expected to occur within minutes, there must be an intracellular pool of FAT/CD36 present within muscle cells from which this protein can be recruited to the sarcolemma. The first evidence for such an intracellular pool of FAT/CD36 within myocytes was obtained from a novel method of fractionate skeletal muscle into membrane fractions from distinct subcellular origin. Using this method, the majority of GLUT4 and about half of FAT/ CD36 were found to be present in a transferrin receptorcontaining fraction, presumably endosomes (Bonen et al. 2000). The onset of contractions resulted in mobilization of both transporters from these endosomal stores to the sarcolemma. Recently by using immunofluorescence microscopy, it has been possible to confirm that within the human vastus lateralis muscle FAT/CD36 is localized both at the sarcolemma and throughout the cytoplasm; the latter site is apparent as a scatter pattern (Keizer et al. 2004). After adapting the fractionation technique to cardiac myocytes, it was also subsequently demonstrated that within these cells under non-stimulatory conditions FAT/CD36 is localized both at the sarcolemma and in intracellular stores (Luiken et al. 2002a).

\section{Contraction-induced transporter translocation in cardiac myocytes}

It can be reasoned that the presence of contractionresponsive intracellular pools of substrate transporters in skeletal muscle is of high physiological importance, because it enables skeletal muscle to acutely increase substrate uptake whenever the metabolic demands suddenly increase. However, the presence of extensive intracellular storage sites for these proteins in nonstimulated cardiac myocytes in suspensions would then be atypical because of the continuous contractile performance of the heart in vivo. Nonetheless, the heart can respond to acute exercise or stress by increasing its pump rate by up to approximately 3 -fold, and it therefore still relies on the presence of intracellular stores of substrate transporters in order to fulfill these conditions, albeit that these stores are probably substantially smaller than those in non-stimulated cardiac myocytes in suspension. 
Despite these considerations, the use of isolated cardiac myocytes has been proved to be suitable for unravelling intracellular events underlying the increase in substrate uptake that occurs with contractile activity. The comparison of subcellular localization of substrate transporters in quiescent and stimulated cardiac myocytes demonstrates that the intrinsic property of the heart is to store a substantial portion of these transporters intracellularly, while the permanent presence of contractile stimulation results in their continuous mobilization. This property would have remained difficult to detect when studying perfused working hearts or performing in vivo measurements, because in these models a higher percentage of transporters would reside at the cell surface, while the intracellular depots would be marginally smaller.

\section{Identification of signalling enzymes involved in contraction-induced substrate transporter translocation}

The biochemistry of muscle contractions has been well characterized, and is known to include $\mathrm{Ca}^{2+}$ oscillations and phosphorylation of proteins of the contractile machinery (Schaub \& Kunz, 1986; Murray et al. 1989). Furthermore, the metabolic demands of contractions favour the activation of stress-sensing cascades. Hence, likely candidates for the contraction-induced translocation of substrate transporters include protein kinase A (PKA), AMP-activated protein kinase (AMPK), members of the protein kinase $\mathrm{C}$ (PKC) family and the extracellular signalregulated kinases.

\section{Protein kinase A}

It is well documented that myocyte contractility is regulated by the intracellular levels of cAMP. The effects of cAMP on contraction are mediated through PKAinduced phosphorylation of proteins involved in myocardial $\mathrm{Ca}^{2+}$ regulation, such as the sarcolemmal L-type $\mathrm{Ca}^{2+}$ channel (Hussain \& Orchard, 1997). Manipulations to raise the intracellular cAMP concentration have an inotropic effect. For example, Kammermeier and co-workers (Rose et al. 1991) have demonstrated that $\beta$-agonists enhance the amplitude of the contraction of isolated cardiac myocytes up to 3-fold. This inotropic action leads to increased energy demands and a drastic rise in $\mathrm{O}_{2}$ consumption.

Information about the effect of $\beta$-agonists on glucose uptake into cardiac myocytes is scarce. However, it has been reported that $\beta$-adrenergic activation does not participate in stress-induced glucose uptake in perfused rat hearts (Egert et al. 1999). In isolated cardiac myocytes isoproterenol has an antagonistic effect on the stimulation of glucose uptake by sulfhydryl reagents (Fischer et al. 1993).

In relation to LCFA uptake, a possible involvement of PKA in contraction-induced FAT/CD36 translocation has been investigated by pharmacologically manipulating the intracellular cAMP level. On the addition of isoproterenol, dibutyryl-cAMP and various phosphodiesterase inhibitors to either quiescent or contracting cardiac myocytes, intracellular cAMP levels rise to variable extents, but are not in any instance associated with an increase in FAT/CD36-mediated
LCFA uptake (Luiken et al. 2002b). Furthermore, electrical stimulation of cardiac myocytes did stimulate LCFA uptake but had no effect on intracellular cAMP (Luiken et al. 2002b).

Hence, it is unlikely that PKA signalling is involved in the stimulation of uptake of glucose and LCFA. However, intracellular cAMP levels do affect the metabolic fate of LCFA, and this influence is dependent on the inotropic action of cAMP causing elevated levels, so that it can only be attained in electrically-stimulated cells. When the intracellular levels of cAMP are elevated in contracting myocytes upon $\beta$-adrenergic stimulation, the rate of LCFA uptake does not change, but LCFA are more efficiently directed into mitochondrial $\beta$-oxidation at the expense of esterification into triacylglycerol stores (Luiken et al. $2002 b$ ). This cAMP-induced channeling of LCFA away from storage into $\beta$-oxidation is in line with the ability of PKA to phosphorylate acetyl-CoA carboxylase (Boone et al. 1999). Phosphorylation of this enzyme then results in its inhibition, causing a fall in intracellular malonyl$\mathrm{CoA}$ and a concomitant deinhibition of the rate-limiting enzyme of mitochondrial $\beta$-oxidation, carnitine palmitoyl transferase 1.

Members of the family of phosphodiesterases not only regulate cAMP, but are also involved in regulation of levels of the second messenger cGMP. The application of cGMP-specific phosphodiesterase inhibitors, which substantially raise the intracellular cGMP levels, has no effect on LCFA uptake by cardiac myocytes (Luiken et al. 2002b). This finding also excludes this second messenger and its downstream signalling targets, such as protein kinase $G$, from being involved in contraction-induced transporter mobilization.

\section{AMP-activated protein kinase}

The ability of AMPK to initiate a catabolic programme in the cell has recently been considered of therapeutic potential when fighting insulin resistance and type 2 diabetes in lipid-accumulating cells (Mauvais-Jarvis et al. 2001). AMPK is activated by an increase in intracellular AMP:ATP (Hardie et al. 1998). An increase in the intracellular concentration of AMP at the expense of ATP can be achieved physiologically by an increase in workload, such as contractile activity, and pharmacologically by mitochondrial inhibitors. The mitochondrial inhibitor oligomycin elevates cardiomyocytic AMP:ATP by 2.5fold, whereas $4 \mathrm{~Hz}$ contractions evoke an increase of 1.9-fold (Luiken et al. 2003). With both manipulations the rise in AMP:ATP has proved to be sufficient to activate AMPK, as assessed by the extent of phosphorylation of its main protein substrate acetyl-CoA carboxylase. Again, oligomycin (8.7-fold increase) is more potent than $4 \mathrm{~Hz}$ contractions (3·3-fold increase; Fig. 1).

The most common tool for studying AMPK is the cellpermeable adenosine analogue 5-aminoimidazole-4-carboxamide-1- $\beta$-D-riboside (AICAR), which on intracellular entry is rapidly phosphorylated to 5-amino-4-imidazolecarboxamide riboside $5^{\prime}$-monophosphate. In turn, 5-amino4-imidazolecarboxamide riboside 5'-monophosphate, like AMP, is able to activate AMPK. The ability of AICAR to 
(a) Control Oligomycin AICAR PMA
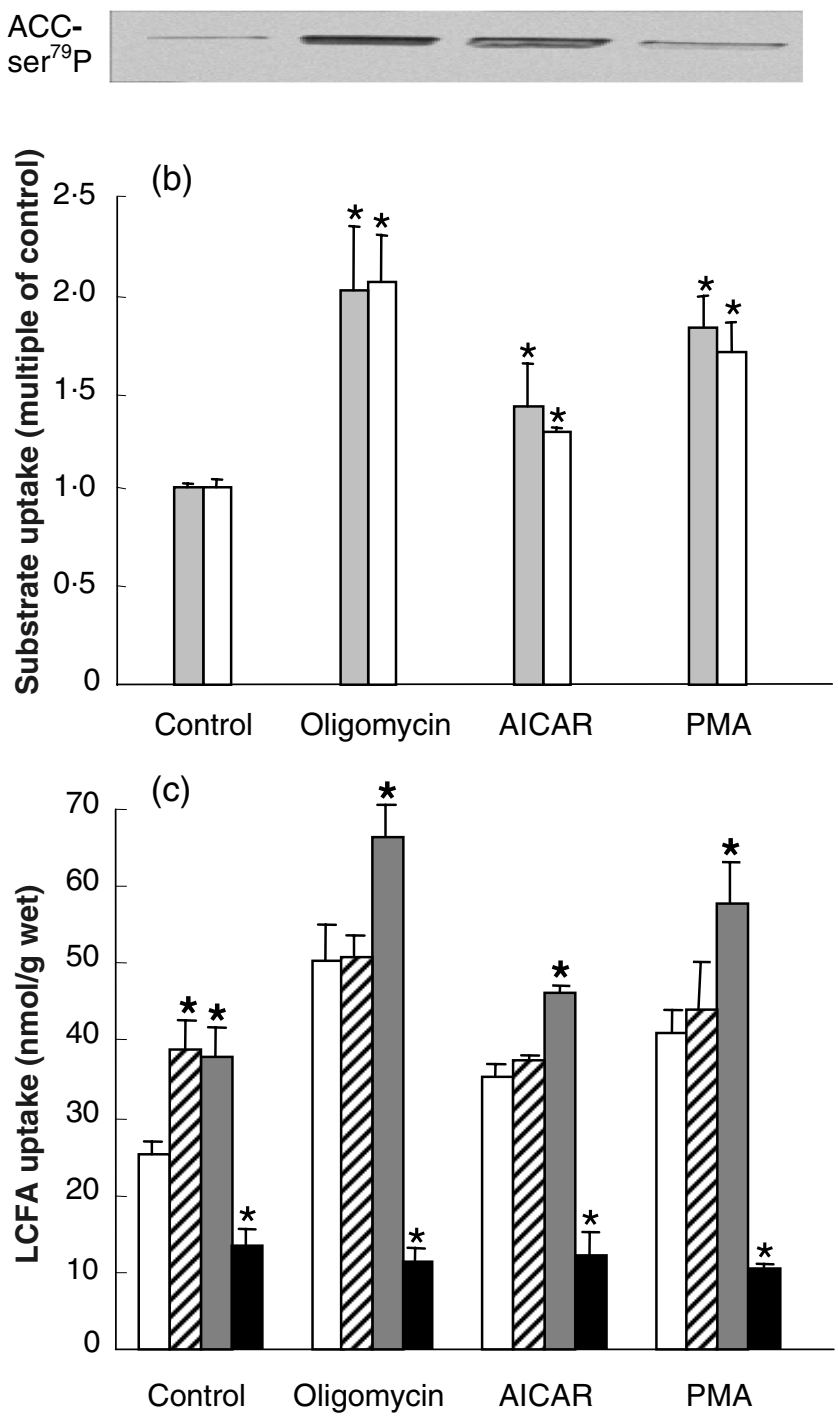

Fig. 1. Effects of oligomycin, 5-aminoimidazole-4-carboxamide1- $\beta$-D-riboside (AICAR) or phorbol 12-myristate 13-acetate (PMA) on AMP-activated protein kinase (AMPK) activation and substrate uptake by cardiac myocytes. Cell suspensions were incubated in the absence of additions (control) or presence of $30 \mu \mathrm{m}$-oligomycin, $1.0 \mathrm{~mm}$-AICAR or $1.0 \mu \mathrm{m}$-PMA for $15 \mathrm{~min}$ before the determination of AMPK activation and substrate uptake. (a) AMPK activation was assessed by its ability to phosphorylate acetyl-CoA carboxylase (ACC) using an antiserum specific for the serine 79-phosphorylated $280 \mathrm{kDa}$ isoform. (b) Uptake of $\left[{ }^{3} \mathrm{H}\right]$ deoxyglucose $(\square)$ and $\left[{ }^{14} \mathrm{C}\right]$ palmitate $(\square)$ were determined simultaneously after $3 \mathrm{~min}$ incubation as pelletable radioactivity. (c) The effect of oligomycin, AICAR or PMA on palmitate uptake was examined in the absence $(\square)$ or presence $(\square)$ of electrical stimulation at $200 \mathrm{~V}$ and $4 \mathrm{~Hz}$ during pre-incubation and incubation, or in the presence of $10 \mathrm{~nm}$ insulin ( $\square$ ) or $0.4 \mathrm{~mm}$-sulfo- $\mathrm{N}$-succinimidyloleate (SSO; $\square$ ) during pre-incubation. Before incubation with radiolabelled substrate, SSO, but not insulin, was removed by washing. Values are means with their standard errors represented by vertical bars. Mean values were significantly different from those for the controls without additions: ${ }^{\star} P<0.05$. (Some of the data are from Luiken et al. 2003.) specifically activate AMPK has promoted its potential use as an anti-diabetic drug (Musi \& Goodyear, 2003; Ruderman et al. 2003). In experiments with suspensions of cardiac myocytes AICAR has proved to be successful in enhancing acetyl-CoA carboxylase phosphorylation (by 5.5-fold; Fig. 1). However, side effects of AICAR on intermediary metabolism have been reported that are unrelated to 5-amino-4-imidazolecarboxamide riboside 5 '-monophosphate formation and, therefore, do not depend on AMPK activation (Corton et al. 1995; Javaux et al. 1995). These non-specific AICAR effects prompted the inclusion in our studies of the adenosine kinase inhibitor 5-iodotubercidin, which prevents 5-amino-4-imidazolecarboxamide riboside 5'-monophosphate formation from AICAR (Samari \& Seglen, 1998). In the presence of this inhibitor AICAR completely loses its ability to phosphorylate acetyl-CoA carboxylase, indicating the involvement of AMPK (Luiken et al. 2003).

Activation of AMPK by either oligomycin or AICAR has substantial effects on substrate utilization by cardiac myocytes. Both these agents stimulate the rate of uptake of both glucose and LCFA, to a similar extent (Fig. 1). In agreement with the notion that 5-amino-4-imidazolecarboxamide riboside $5^{\prime}$-monophosphate is a weaker activator of AMPK than AMP (Javaux et al. 1995), the effect of AICAR on glucose and LCFA uptake is smaller than that of oligomycin (Luiken et al. 2003). While the stimulatory effect of mitochondrial inhibitors and AICAR on glucose uptake has been attributed to translocation of GLUT4 from intracellular stores to the sarcolemma (Wheeler et al. 1994; Russell et al. 1999), the stimulatory effect on LCFA uptake is a novel observation. The ability of sulfo- $N$ succinimidyloleate to block oligomycin- and AICARinduced LCFA uptake suggests the involvement of FAT/ CD36 in the stimulatory action of both agents. The nonadditivity of either of these agents with $4 \mathrm{~Hz}$ contractions on LCFA uptake (Fig. 1) indicates strongly that both agents exert their effect on LCFA uptake via the same mechanism, i.e. a rapid translocation of FAT/CD36 from intracellular membrane stores to the sarcolemma. Hence, elevation of AMP:ATP and subsequent activation of AMPK are necessary events for contractions to stimulate FAT/CD36 translocation and, consequently, LCFA uptake. On the other hand, additivity in relation to LCFA uptake of insulin and oligomyin and of insulin and AICAR indicates that insulin signalling is not involved in the effect of contractions on LCFA uptake (Luiken et al. 2003). Accordingly, it was found that the phosphatidylinositol-3 kinase inhibitor wortmannin is ineffective in inhibiting the effect of either oligomycin or AICAR on contractions (Luiken et al. 2003).

Since AMPK has been demonstrated to be involved in the activation of not only FAT/CD36 (the present study) but also of another component of the LCFA metabolic machinery, i.e. carnitine palmitoyltransferase 1 (Ruderman et al. 1999), the simultaneous stimulation of LCFA uptake and oxidation would be metabolically efficient because this process allows the extra incoming LCFA during contractions to be preferentially used for energy production. 


\section{Protein kinase $C$}

The PKC are a family of serine/threonine kinases consisting of at least twelve isoforms. Based on the ability of these isoforms to be activated by $\mathrm{Ca}^{2+}$ or by the lipid metabolite diacylglycerol, they are subdivided into three classes: conventional (activation by both $\mathrm{Ca}^{2+}$ and diacylglycerol), novel (activation by only diacylglycerol) and atypical PKC (activation by neither $\mathrm{Ca}^{2+}$ nor diacylglycerol). At the protein level, at least four isoforms appear to be present in cardiac myocytes, notably the conventional PKC $\alpha$, the novel PKC $\delta$ and PKCE and the atypical PKCל (Disatnik et al. 1994; Puceat et al. 1994; Clerk et al. 1995; Goldberg et al. 1997). PKC are implicated in various cellular processes, including the regulation of contractile function, but there is no available information about the involvement of the different isoforms (Steinberg et al. 1995). The phorbol ester phorbol 12-myristate 13-acetate (PMA) is a cell-permeable diacylglycerol analogue that is commonly used to pharmacologically activate conventional and novel PKC. In relation to substrate utlilization, PMA has been shown to induce the translocation of GLUT4 in adipocytes, leading to enhanced glucose uptake (Vogt et al. 1991; Nishimura
\& Simpson, 1994). Adding PMA to cardiac myocytes has confirmed the involvement of PKC in the stimulation of glucose uptake (Fig. 1(b)). In this study, it is shown for the first time that PMA also stimulates LCFA uptake by cardiac myocytes. It is likely that FAT/CD36 is involved in this novel action of PMA because in the presence of sulfo-N-succinimidyloleate, PMA completely loses its ability to stimulate LCFA uptake (Fig. 1(c)). Furthermore, PMA inducible LCFA uptake is non-additive to the effect of contractions and additive to that of insulin. In this, the action of PMA on LCFA uptake resembles those of AICAR and oligomycin (Fig. 1(c)), suggesting that PMA acts on the contraction signalling pathway but not on insulin signalling. Activation of contraction signalling is then expected to result in FAT/CD36 translocation from intracellular stores to the sarcolemma. However, in contrast to contractions and oligomycin, PMA has no effect on AMPK activation (Fig. 1(a)). These observations lead to the conclusion that conventional and/or novel PKC are involved in contraction-induced recruitment of GLUT4 and FAT/CD36 from intracellular stores to the sarcolemma (Fig. 2). Interestingly, it was observed that in the presence of specific PKC inhibitors, activation of AMPK does not result in stimulation of uptake of LCFA and of glucose

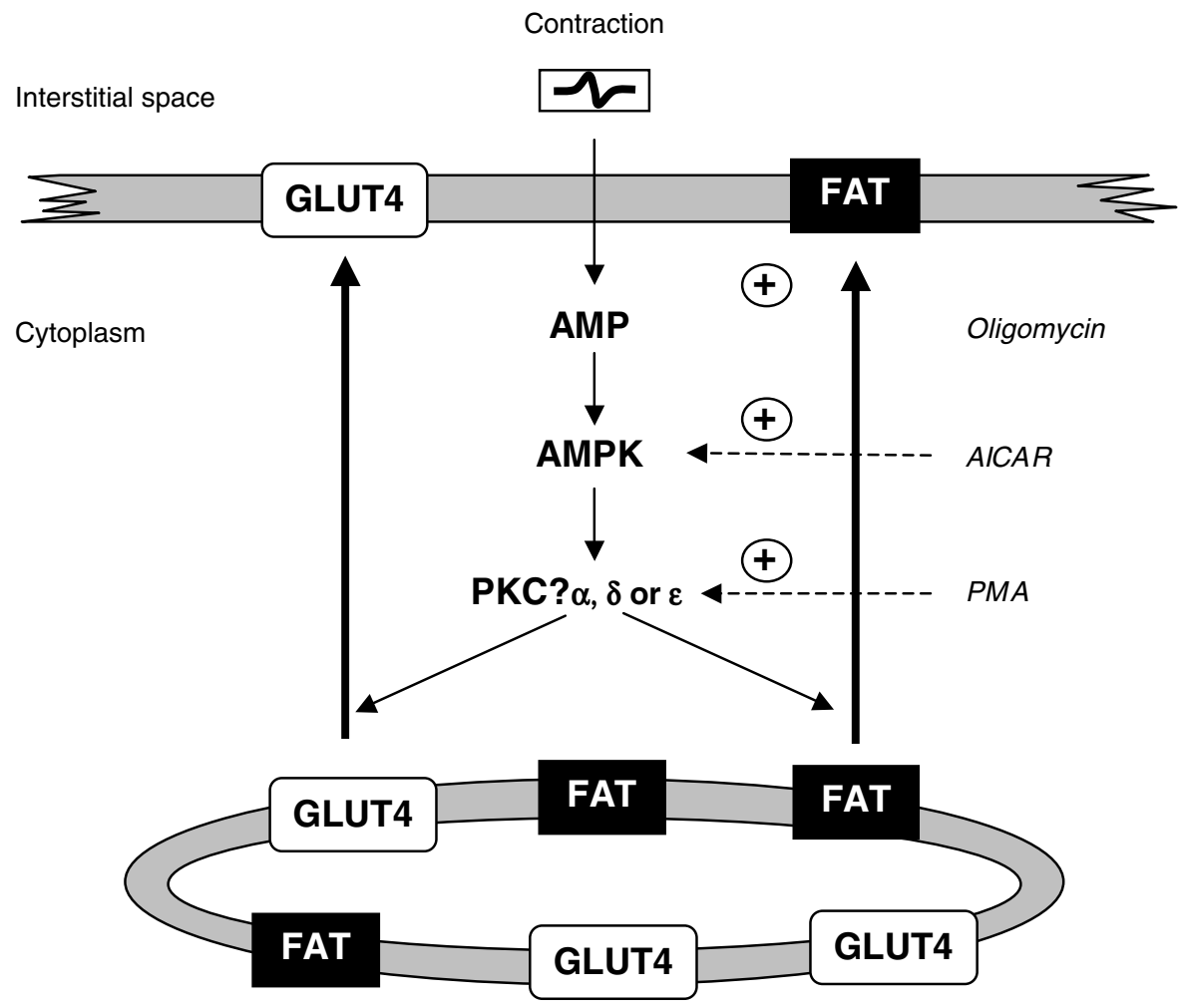

Fig. 2. Schematic presentation of the main signalling components involved in the mobilization of fatty acid translocase (FAT)/CD36 and GLUT4 by contractions in cardiac myocytes. Cellular contractions induce ATP utilization concomitant with an elevation of intracellular AMP. This increase in AMP causes activation of AMP-activated protein kinase (AMPK), which in turn activates one (or more) cardiac protein kinase $C(P K C)$ isoform(s), specifically the conventional $\mathrm{PKC} \alpha$, or the novel PKC $\delta$ and PKC $\varepsilon$. The signal downstream of these PKC isoforms is as yet obscure, but it results in recruitment of both GLUT4 and FAT/CD36 from an intracellular storage compartment. Pharmacological activation of contraction-induced signalling by oligomycin, 5-aminoimidazole-4-carboxamide-1- $\beta$-D-riboside (AICAR) and phorbol 12-myristate 13acetate (PMA) also leads to the simultaneous recruitment of GLUT4 and FAT/CD36 (see Fig. 1). 
(JJFP Luiken, J Willems, SLM Coort and JFC Glatz, unpublished results). Hence, the PKC should be positioned downstream rather than upstream of AMPK. This notion would be of interest to signal transduction research, because signalling downstream of AMPK has not been clearly identified (Hardie \& Hawley, 2001; Musi \& Goodyear, 2003). This putative positioning of PKC in contraction signalling requires the activation of a lipid metabolizing enzyme by AMPK and the subsequent formation of diacylglycerol, the endogenous activator of conventional and novel PKC.

Interestingly, in addition to PMA-inducible $\mathrm{PKC}$, the PMA-insensitive PKC $\zeta$ has also been implicated in GLUT4 translocation. There is accumulating evidence that $\mathrm{PKC} \zeta$ is located downstream of insulin receptor substrates and phosphatidylinositol-3 kinase, and is involved in the translocation of GLUT4 in response to insulin (Farese, 2002). Its role in contraction-inducible GLUT4 has only recently been investigated (Chen et al. 2002). In mouse skeletal muscle both treadmill exercise and AICAR have been shown to induce activation of $\mathrm{PKC} \zeta$. Moreover, myristoylated PKC $\zeta$ pseudosubstrate can block AICARinduced glucose uptake by L6 myotubes (Chen et al. 2002). To finally pinpoint $\mathrm{PKC} \zeta$ to contraction signalling, additional evidence is required to elucidate whether this AICAR effect is mediated through AMPK. If PKC $\zeta$ is involved in contraction-induced substrate uptake it should operate in concert with one (or more) PMA-inducible $\mathrm{PKC}$. Where $\mathrm{PKC} \zeta$ is positioned relative to the PMAinducible PKC along the contraction signalling pathway is a topic for future research.

\section{Extracellular signal-regulated kinases}

Contractile performance of skeletal muscle has been shown to activate extracellular signal-regulated kinase by phosphorylation. However, inhibition of extracellular signal-regulated kinase phosphorylation by a mitogenactivated protein kinase kinase inhibitor does not inhibit contraction-induced glucose uptake in isolated muscle strips (Hayashi et al. 1999) or in perfused skeletal muscle (Wojtaszewski et al. 1999). Thus, it is apparent that this signalling enzyme is not involved in stimulation of glucose uptake into heart when the workload is increased. However, the involvement of extracellular signal-regulated kinase in the regulation of LCFA uptake into heart and skeletal muscle has not yet been investigated.

\section{Concluding remarks}

Cardiac diseases have often been linked with a change in substrate preference. Thus, cardiac hypertrophy is associated with a shift in substrate utilization from LCFA to glucose (Van Bilsen et al. 1997; van der Vusse et al. 2000), while, on the other hand, in diabetes the heart preferentially uses LCFA at the expense of glucose (Rodrigues et al. 1998; Shulman, 2000; Unger \& Orci, 2001). Since it appears that in the utilization of both substrates by the heart their uptake across the sarcolemma appears to be a rate-governing step (Glatz et al. 2002), it is of pivotal importance to investigate the mechanism of regulation of cardiac LCFA uptake in comparison with that of glucose uptake. Based on the data reported earlier, it can be concluded that the intracellular transduction pathways leading to stimulation of both glucose and LCFA uptake are identical to a large extent, as it has been shown that contraction-inducible recruitment of both GLUT4 and FAT/CD36 are dependent on activation of AMPK as well as on activation of conventional or novel PKC. The participation of the same signalling enzymes in the stimulation of both glucose and LCFA uptake may suggest that a single subcellular compartment is utilized for intracellular storage of both GLUT4 and FAT/CD36. On the other hand, it is equally possible that both transporters are stored in distinct compartments and that downstream of the PKC the contraction signal branches off into two pathways leading to separate GLUT4 and FAT/CD36 mobilization. This latter possibility would allow manipulation of substrate preference by the heart. Finally, although not yet proven, it is anticipated that AMPK and PKC are both also involved in the enhancement of the uptake of substrate by contracting skeletal muscle.

\section{Acknowledgements}

This work is supported by the Netherlands Heart Foundation (grant 2000.156) and by the Heart \& Stroke Foundation of Ontario, Canada. Antibody MO25 was kindly provided by Dr N. N. Tandon, Thrombosis and Vascular Biology Laboratory, Otsuka America Pharmaceutical Inc., Rockville, MD, USA. J.J.F.P.L. is the recipient of a VIDIInnovational Research Grant from the Netherlands Organisation for Scientific Research (ZonMw grant no. 016.036.305). A.B. holds a Canada Research Chair in Metabolism and Health. J.F.C.G. is Netherlands Heart Foundation Professor of Cardiac Metabolism.

\section{References}

Bonen A, Luiken JJ, Arumugam Y, Glatz JF \& Tandon NN (2000) Acute regulation of fatty acid uptake involves the cellular redistribution of fatty acid translocase. Journal of Biological Chemistry 275, 14501-14508.

Bonen A, Luiken JJ \& Glatz JF (2002) Regulation of fatty acid transport and membrane transporters in health and disease. Molecular and Cellular Biochemistry 239, 181-192.

Boone AN, Rodrigues B \& Brownsey RW (1998) Multiple-site phosphorylation of the $280 \mathrm{kDa}$ isoform of acetyl-CoA carboxylase in rat cardiac myocytes: evidence that cAMP-dependent protein kinase mediates effects of beta-adrenergic stimulation. Biochemical Journal 341, 347-354.

Chen HC, Bandyopadhyay G, Sajan MP, Kanoh Y, Standaert M, Farese RV Jr \& Farese RV (2002) Activation of the ERK pathway and atypical protein kinase $\mathrm{C}$ isoforms in exercise- and aminoimidazole-4-carboxamide-1-beta-D-riboside (AICAR)stimulated glucose transport. Journal of Biological Chemistry 277, 23554-23562.

Clerk A, Bogoyevitch MA, Fuller SJ, Lazou A, Parker PJ \& Sugden PH (1995) Expression of protein kinase C isoforms during cardiac ventricular development. American Journal of Physiology 269, H1087-H1097. 
Corton JM, Gillespie JG, Hawley SA \& Hardie DG (1995) 5-Aminoimidazole-4-carboxamide ribonucleoside. A specific method for activating AMP-activated protein kinase in intact cells? European Journal of Biochemistry 229, 558-565.

Cushman SW, Goodyear LJ, Pilch PF, Ralston E, Galbo H, Ploug T, Kristiansen S \& Klip A (1998) Molecular mechanisms involved in GLUT4 translocation in muscle during insulin and contraction stimulation. Advances in Experimental Medicine and Biology 441, 63-71.

Disatnik MH, Buraggi G \& Mochly-Rosen D (1994) Localization of protein kinase $\mathrm{C}$ isozymes in cardiac myocytes. Experimental Cell Research 210, 287-297.

Egert S, Nguyen N \& Schwaiger M (1999) Contribution of alpha-adrenergic and beta-adrenergic stimulation to ischemiainduced glucose transporter (GLUT) 4 and GLUT1 translocation in the isolated perfused rat heart. Circulation Research 84, $1407-1415$.

Farese RV (2002) Function and dysfunction of aPKC isoforms for glucose transport in insulin-sensitive and insulin-resistant states. American Journal of Physiology 283, E1-E11.

Fischer Y, Rose H, Thomas J, Deuticke B \& Kammermeier H (1993) Phenylarsine oxide and hydrogen peroxide stimulate glucose transport via different pathways in isolated cardiac myocytes. Biochimica et Biophysica Acta 1153, 97-104.

Glatz JF, Bonen A \& Luiken JJ (2002) Exercise and insulin increase muscle fatty acid uptake by recruiting putative fatty acid transporters to the sarcolemma. Current Opinion in Clinical Nutrition and Metabolic Care 5, 365-370.

Goldberg M, Zhang HL \& Steinberg SF (1997) Hypoxia alters the subcellular distribution of protein kinase $\mathrm{C}$ isoforms in neonatal rat ventricular myocytes. Journal of Clinical Investigation 99, 55-61.

Hamilton JA (1998) Fatty acid transport: difficult or easy? Journal of Lipid Research 39, 467-481.

Hardie DG, Carling D \& Carlson M (1998) The AMPactivated/SNF1 protein kinase subfamily: metabolic sensors of the eukaryotic cell? Annual Review of Biochemistry 67, 821-855.

Hardie DG \& Hawley SA (2001) AMP-activated protein kinase: the energy charge hypothesis revisited. Bioessays 23, 1112-1119.

Hayashi T, Hirshman MF, Dufresne SD \& Goodyear LJ (1999) Skeletal muscle contractile activity in vitro stimulates mitogenactivated protein kinase signaling. American Journal of Physiology 277, C701-C707.

Hussain M \& Orchard CH (1997) Sarcoplasmic reticulum $\mathrm{Ca}^{2+}$ content, L-type $\mathrm{Ca}^{2+}$ current and the $\mathrm{Ca}^{2+}$ transient in rat myocytes during beta-adrenergic stimulation. Journal of Physiology (London) 505, 385-402.

Javaux F, Vincent MF, Wagner DR \& van den Berghe G (1995) Cell-type specificity of inhibition of glycolysis by 5-amino4-imidazolecarboxamide riboside. Lack of effect in rabbit cardiomyocytes and human erythrocytes, and inhibition in FTO-2B rat hepatoma cells. Biochemical Journal 305, 913-919.

Keizer HA, Schaart G, Tandon NN, Glatz JFC \& Luiken JJFP (2004) Subcellular immunolocalisation of fatty acid translocase (FAT)/CD36 in human type-1 and type-2 skeletal muscle fibres. Histochemical Cell Biology 121, 101-107.

Kones RJ \& Phillips JH (1975) Insulin: fundamental mechanism of action and the heart. Cardiology 60, 280-303.

Luiken JJ, Coort SL, Willems J, Coumans WA, Bonen A, van der Vusse GJ \& Glatz JF (2003) Contraction-induced fatty acid translocase/CD36 translocation in rat cardiac myocytes is mediated through AMP-activated protein kinase signaling. Diabetes 52, 1627-1634.

Luiken JJ, Koonen DP, Willems J, Zorzano A, Becker C, Fischer Y, Tandon NN, Van Der Vusse GJ, Bonen A \& Glatz JF (2002a) Insulin stimulates long-chain fatty acid utilization by rat cardiac myocytes through cellular redistribution of FAT/ CD36. Diabetes 51, 3113-3119.

Luiken JJ, Willems J, Coort SL, Coumans WA, Bonen A, Van Der Vusse GJ \& Glatz JF (2002b) Effects of cAMP modulators on long-chain fatty-acid uptake and utilization by electrically stimulated rat cardiac myocytes. Biochemical Journal 367, 881-887.

Luiken JJ, Willems J, van der Vusse GJ \& Glatz JF (2001) Electrostimulation enhances FAT/CD36-mediated long-chain fatty acid uptake by isolated rat cardiac myocytes. American Journal of Physiology 281, E704-E712.

Mauvais-Jarvis F, Andreelli F, Hanaire-Broutin H, Charbonnel B \& Girard J (2001) Therapeutic perspectives for type 2 diabetes mellitus: molecular and clinical insights. Diabetes Metabolism 27, 415-423.

Murray KJ, Reeves ML \& England PJ (1989) Protein phosphorylation and compartments of cyclic AMP in the control of cardiac contraction. Molecular and Cellular Biochemistry 89. $175-179$.

Musi N \& Goodyear LJ (2003) AMP-activated protein kinase and muscle glucose uptake. Acta Physiologica Scandinavica 178, 337-345.

Nishimura H \& Simpson IA (1994) Staurosporine inhibits phorbol 12-myristate 13-acetate- and insulin-stimulated translocation of GLUT1 and GLUT4 glucose transporters in rat adipose cells. Biochemical Journal 302, 271-277.

Ploug T \& Ralston E (2002) Exploring the whereabouts of GLUT4 in skeletal muscle (review). Molecular Membrane Biology 19, 39-49.

Puceat M, Hilal-Dandan R, Strulovici B, Brunton LL \& Brown JH (1994) Differential regulation of protein kinase C isoforms in isolated neonatal and adult rat cardiomyocytes. Journal of Biological Chemistry 269, 16938-16944.

Rodrigues B, Cam MC \& McNeill JH (1998) Metabolic disturbances in diabetic cardiomyopathy. Molecular and Cellular Biochemistry 180, 53-57.

Rose H, Strotmann KH, Popping S, Fischer Y, Kulsch D \& Kammermeier H (1991) Simultaneous measurement of contraction and oxygen consumption in cardiac myocytes. American Journal of Physiology 261, H1329-H1334.

Ruderman NB, Park H, Kaushik VK, Dean D, Constant S, Prentki M \& Saha AK (2003) AMPK as a metabolic switch in rat muscle, liver and adipose tissue after exercise. Acta Physiologica Scandinavica 178, 435-442.

Ruderman NB, Saha AK, Vavvas D \& Witters LA (1999) Malonyl-CoA, fuel sensing, and insulin resistance. American Journal of Physiology 276, E1-E18.

Russell RR 3rd, Bergeron R, Shulman GI \& Young LH (1999) Translocation of myocardial GLUT-4 and increased glucose uptake through activation of AMPK by AICAR. American Journal of Physiology 277, H643-H649.

Samari HR \& Seglen PO (1998) Inhibition of hepatocytic autophagy by adenosine, aminoimidazole-4-carboxamide riboside, and N6-mercaptopurine riboside. Evidence for involvement of AMP-activated protein kinase. Journal of Biological Chemistry 273, 23758-23763.

Schaub MC \& Kunz B (1986) Regulation of contraction in cardiac and smooth muscles. Journal of Cardiovascular Pharmacology 8, Suppl. 8, S117-S123.

Shulman GI (2000) Cellular mechanisms of insulin resistance. Journal of Clinical Investigation 106, 171-176.

Steinberg SF, Goldberg M \& Rybin VO (1995) Protein kinase C isoform diversity in the heart. Journal of Molecular and Cellular Cardiology 27, 141-153.

Till M, Kolter T \& Eckel J (1997) Molecular mechanisms of contraction-induced translocation of GLUT4 in isolated 
cardiomyocytes. American Journal of Cardiology 80, 85A89A.

Unger RH \& Orci L (2001) Diseases of liporegulation: new perspective on obesity and related disorders. FASEB Journal 15, 312-321.

Van Bilsen M, de Vries JE \& Van der Vusse GJ (1997) Longterm effects of fatty acids on cell viability and gene expression of neonatal cardiac myocytes. Prostaglandins, Leukotrienes and Essential Fatty Acids 57, 39-45.

van der Vusse GJ, Glatz JF, Stam HC \& Reneman RS (1992)

Fatty acid homeostasis in the normoxic and ischemic heart. Physiological Reviews 72, 881-940.

van der Vusse GJ, van Bilsen M \& Glatz JF (2000) Cardiac fatty acid uptake and transport in health and disease. Cardiovascular Research 45, 279-293.
Vogt B, Mushack J, Seffer E \& Haring HU (1991) The translocation of the glucose transporter sub-types GLUT1 and GLUT4 in isolated fat cells is differently regulated by phorbol esters. Biochemical Journal 275, 597-600.

Wheeler TJ, Fell RD \& Hauck MA (1994) Translocation of two glucose transporters in heart: effects of rotenone, uncouplers, workload, palmitate, insulin and anoxia. Biochimica et Biophysica Acta 1196, 191-200.

Wojtaszewski JF, Lynge J, Jakobsen AB, Goodyear LJ \& Richter EA (1999) Differential regulation of MAP kinase by contraction and insulin in skeletal muscle: metabolic implications. American Journal of Physiology 277, E724-E732.

Zorzano A, Fandos C \& Palacin M (2000) Role of plasma membrane transporters in muscle metabolism. Biochemical Journal 349, 667-688. 